\title{
Beta-Cauchy Distribution: Some Properties and Applications
}

\author{
Etaf Alshawarbeh \\ Department of Mathematics, Central Michigan University \\ Mount Pleasant, MI 48859, USA \\ Email:alsha1e@cmich.edu \\ Felix Famoye \\ Department of Mathematics, Central Michigan University \\ Mount Pleasant, MI 48859, USA \\ Email: famoy1kf@cmich.edu \\ Carl Lee \\ Department of Mathematics, Central Michigan University \\ Mount Pleasant, MI 48859, USA \\ Email:lee1c@cmich.edu \\ Received 8 December 2012 \\ Accepted 9 August 2013
}

\begin{abstract}
Some properties of the four-parameter beta-Cauchy distribution such as the mean deviation and Shannon's entropy are obtained. The method of maximum likelihood is proposed to estimate the parameters of the distribution. A simulation study is carried out to assess the performance of the maximum likelihood estimates. The usefulness of the new distribution is illustrated by applying it to three empirical data sets and comparing the results to some existing distributions. The beta-Cauchy distribution is found to provide great flexibility in modeling symmetric and skewed heavy-tailed data sets.
\end{abstract}

Keywords and Phrases: Beta family, mean deviation, entropy, maximum likelihood estimation.

\section{Introduction}

Eugene et al. ${ }^{1}$ introduced a class of new distributions using beta distribution as the generator and pointed out that this can be viewed as a family of generalized distributions of order statistics. Jones $^{2}$ studied some general properties of this family. This class of distributions has been referred to as "beta-generated distributions". ${ }^{3}$ This family of distributions provides some flexibility in modeling data sets with different shapes. Let $F(x)$ be the cumulative distribution function (CDF) of a random variable $X$, then the CDF $G(x)$ for the "beta-generated distributions" is given by

$$
G(x)=[B(\alpha, \beta)]^{-1} \int_{0}^{F(x)} t^{\alpha-1}(1-t)^{\beta-1} d t, \quad 0<\alpha, \beta<\infty,
$$

where $B(\alpha, \beta)=\Gamma(\alpha) \Gamma(\beta) / \Gamma(\alpha+\beta)$. The corresponding probability density function for the beta family of distributions is

$$
g(x)=G^{\prime}(x)=[B(\alpha, \beta)]^{-1}[F(x)]^{\alpha-1}[1-F(x)]^{\beta-1} F^{\prime}(x) .
$$


Eugene et al. ${ }^{1}$ investigated the beta-normal distribution where they presented some properties of the distribution and highlighted its ability to model skewed unimodal data as well as bimodal data. From the beta-family members which have been studied, the shape parameters $\alpha$ and $\beta$ were able to model different shapes by controlling skewness and the tail weight. The ability of the beta-family to model skewed and heavy tailed data as well as bimodal data, in some cases, has inspired studying many beta-family distributions. For a review of beta family distributions, see Ref. 4.

Alshawarbeh et al. ${ }^{5}$ defined the four parameter beta-Cauchy distribution by taking $F(x)$ in Eq. (1) to be the CDF of the Cauchy distribution. The probability density function $g(x)$ for the beta-Cauchy distribution is given by

$$
g(x)=\frac{\lambda}{\pi B(\alpha, \beta)}\left(\frac{1}{2}+\frac{1}{\pi} \arctan \left(\frac{x-\theta}{\lambda}\right)\right)^{\alpha-1}\left(\frac{1}{2}-\frac{1}{\pi} \arctan \left(\frac{x-\theta}{\lambda}\right)\right)^{\beta-1} \frac{1}{\lambda^{2}+(x-\theta)^{2}},
$$

where $-\infty<x<\infty, 0<\alpha, \beta, \lambda<\infty,-\infty<\theta<\infty$.

Alshawarbeh et al. ${ }^{5}$ derived various properties of beta-Cauchy distribution including the non-central moments and the hazard function and also showed that the distribution is unimodal. The parameters $\alpha$ and $\beta$ are shape parameters that control the skewness and kurtosis. The parameter $\theta$ is a location parameter while $\lambda$ is a scale parameter that stretches out or shrinks the distribution. In this article, we derive some additional properties of the beta-Cauchy distribution and discuss maximum likelihood estimation of the parameters. Unlike Cauchy distribution, which is rarely applied to model data, the beta-Cauchy distribution is capable of modeling various shapes of data distributions. To illustrate the flexibility of the beta-Cauchy distribution, we analyze three real data sets and compare the fit with some existing distributions in the beta family. In these applications, the beta-Cauchy performs better than the other distributions in the beta family.

In Sec. 2, we derive some properties of the beta-Cauchy distribution such as the quantile function, the mean deviation and the Shannon's entropy. In Sec. 3, we address the maximum likelihood estimation of the parameters. A simulation study is carried out in Sec. 4 to examine the performance of the maximum likelihood estimates. In Sec. 5, the flexibility of the beta-Cauchy distribution is demonstrated through its applications to real data sets and its comparison to other betafamily distributions. Conclusions are given in Sec. 6.

\section{Some Properties of Beta-Cauchy Distribution}

\subsection{Quantile function}

In this sub-section, we derive the quantile function for the beta-Cauchy random variable.

Lemma 1. The quantile function, $Q(u)$, of the beta-Cauchy random variable is given by

$$
x=Q(u)=\theta+\lambda \tan \left(\pi Q_{\alpha, \beta}(u)-\pi / 2\right),
$$

where $Q_{\alpha, \beta}(u)$, is the quantile function of the beta distribution.

Proof. If $Y$ is a beta random variable with parameters $\alpha$ and $\beta$, then the random variable

$$
X=\theta+\lambda \tan (\pi Y-\pi / 2),
$$

follows a beta-Cauchy distribution in Eq. (3) [see Theorem 1 in Ref. 5]. By using Eq. (5), the beta-Cauchy quantile function can be computed from the beta quantile function as

$$
x=Q(u)=\theta+\lambda \tan \left(\pi Q_{\alpha, \beta}(u)-\pi / 2\right),
$$

where $Q_{\alpha, \beta}(u)$ is the quantile function of the beta distribution. $Q_{\alpha, \beta}(u)$ can be computed by using statistical software 
like SAS or it can be calculated from the inverse of the incomplete beta function as given in Ref. 6 .

Alshawarbeh et $a l^{5}$ showed that the $k^{\text {th }}$ moment of beta-Cauchy distribution exists when $\alpha>k$ and $\beta>k$. The authors provided a table for the skewness and kurtosis for beta-Cauchy distribution for various values of $\alpha$ and $\beta$ when $\theta=0$ and $\lambda=1$. The skewness and kurtosis were based on the moments. Thus, the kurtosis exists only when both $\alpha$ and $\beta$ are greater than 4. In view of this, alternative measures for skewness and kurtosis, based on quantile functions, are more appropriate for beta-Cauchy distribution. The measure of skewness $S$ defined by Galton ${ }^{7}$ and the measure of kurtosis $M$ defined by Moors ${ }^{8}$ are based on quantile functions and they are defined as

$$
S=\frac{Q(6 / 8)-2 Q(4 / 8)+Q(2 / 8)}{Q(6 / 8)-Q(2 / 8)} \text { and } M=\frac{Q(7 / 8)-Q(5 / 8)+Q(3 / 8)-Q(1 / 8)}{Q(6 / 8)-Q(2 / 8)} \text {. }
$$

For some values of $\alpha$ and $\beta$, the values of Galton's skewness and Moors' kurtosis are computed and presented in Table 1.

Table 1. Galton's skewness and Moors' kurtosis for some values of $\alpha$ and $\beta(\theta=0, \lambda=1)$

\begin{tabular}{|ll|cc|cc|cc|}
\hline \multicolumn{2}{|c|}{ Actual values } & \multicolumn{3}{|c|}{} & \multicolumn{2}{|c|}{ Actual values } & \multicolumn{2}{l|}{} \\
$\alpha$ & $\beta$ & Skewness & Kurtosis & $\alpha$ & $\beta$ & Skewness & Kurtosis \\
\hline 0.5 & 0.5 & 0.0 & 3.7848 & 4.0 & 0.5 & 0.6752 & 4.0577 \\
& 1.0 & -0.5412 & 3.7699 & & 1.0 & 0.4298 & 2.0862 \\
& 2.0 & -0.6513 & 3.9984 & & 2.0 & 0.1978 & 1.5227 \\
& 4.0 & -0.6752 & 4.0577 & & 4.0 & 0.0 & 1.3656 \\
& 7.0 & -0.6803 & 4.0705 & & 7.0 & -0.1109 & 1.3573 \\
& 10.0 & -0.6816 & 4.0736 & & 10.0 & -0.1567 & 1.3678 \\
\hline 1.0 & 0.5 & 0.5412 & 3.7699 & 7.0 & 0.5 & 0.6803 & 4.0705 \\
& 1.0 & 0.0 & 2.0 & & 1.0 & 0.4597 & 2.1216 \\
& 2.0 & -0.3185 & 1.9834 & & 2.0 & 0.2740 & 1.5608 \\
& 4.0 & -0.4298 & 2.0862 & & 4.0 & 0.1109 & 1.3573 \\
& 7.0 & -0.4597 & 2.1216 & & 7.0 & 0.0 & 1.3051 \\
& 10.0 & -0.4678 & 2.1316 & & 10.0 & -0.0554 & 1.2991 \\
\hline 2.0 & 0.5 & 0.6513 & 3.9984 & 10.0 & 0.5 & 0.6816 & 4.0736 \\
& 1.0 & 0.3185 & 1.9834 & & 1.0 & 0.4678 & 2.1316 \\
& 2.0 & 0.0 & 1.5322 & & 2.0 & 0.2988 & 1.5774 \\
& 4.0 & -0.1978 & 1.5227 & & 4.0 & 0.1567 & 1.3678 \\
& 7.0 & -0.2740 & 1.5608 & & 7.0 & 0.0554 & 1.2991 \\
& 10.0 & -0.2988 & 1.5774 & & 10.0 & 0.0 & 1.2825 \\
\hline
\end{tabular}

The following are some observations based on Table 1: When $\alpha>\beta$ (or $\alpha<\beta$ ), the beta-Cauchy distribution is positively (or negatively) skewed. For fixed value of $\alpha$, the skewness is a decreasing function of $\beta$; the kurtosis is a decreasing function of $\beta$ when $\alpha>\beta$; and the kurtosis first decrease and then increase as $\beta$ increases when $\alpha<\beta$. For fixed $\beta$, the skewness is an increasing function of $\alpha$; the kurtosis is a decreasing function of $\alpha$ when $\alpha<\beta$; and the kurtosis first decrease and then increase as $\alpha$ increases when $\alpha>\beta$.

By using the quantile function in Eq. (4) for values of $\alpha$ and $\beta$ between 0.2 and 5 and by setting $\theta=0$ and $\lambda=1$, we compute the values of $S$ and $M$. The graphs for the skewness and kurtosis are presented in Fig. 1. 

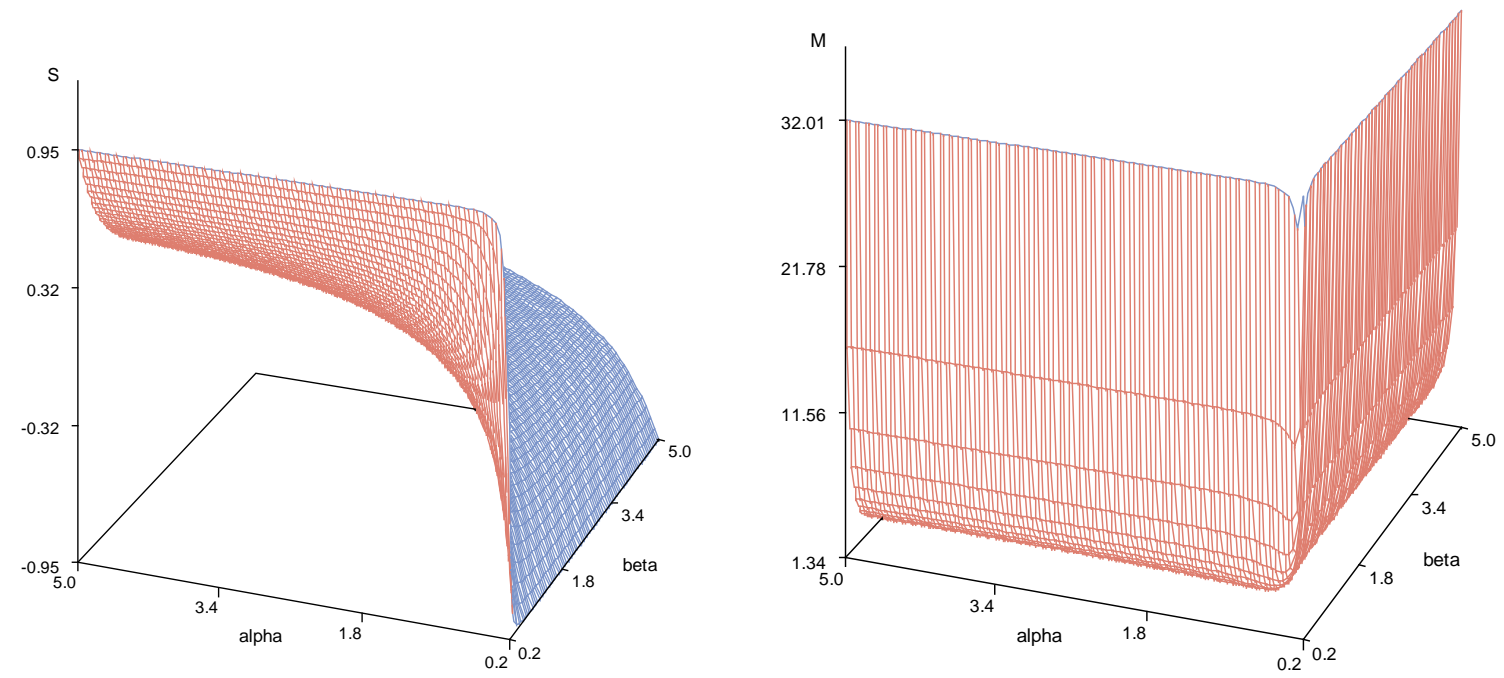

Fig. 1. Galton's skewness $(S)$ and Moors' kurtosis $(M)$ for the beta-Cauchy distribution

\subsection{The mean deviation}

The deviation from the mean or deviation from the median can be used as a measure of spread in a population. Let $X$ be a beta-Cauchy random variable with mean $\mu$ and median $m$. The mean deviation from the mean is defined as

$$
\begin{aligned}
E(|X-\mu|) & =\int_{-\infty}^{\infty}|x-\mu| g(x) d x \\
= & 2 \int_{-\infty}^{\mu}(\mu-x) g(x) d x+\int_{-\infty}^{\infty}(x-\mu) g(x) d x=2 \mu G(\mu)-2 \int_{-\infty}^{\mu} x g(x) d x,
\end{aligned}
$$

where $G(\mu)=\int_{-\infty}^{\mu} g(x) d x$.

Similarly, the mean deviation from the median can be defined as

$$
\begin{aligned}
E(|X-m|)= & \int_{-\infty}^{\infty}|x-m| g(x) d x \\
& =2 \int_{-\infty}^{m}(m-x) g(x) d x+\int_{-\infty}^{\infty}(x-m) g(x) d x=\mu-2 \int_{-\infty}^{m} x g(x) d x .
\end{aligned}
$$

$G(\mu)$ can be calculated from Eq. (1) and the median $m$ can be calculated directly from Eq. (4) by setting $u=1 / 2$.

The next lemma gives a formula for the mean deviation from the mean and the mean deviation from the median for the beta-Cauchy distribution taking into consideration the existence condition for the mean that both $\alpha$ and $\beta$ are greater than 1 [see Theorem 3 in Ref. 5].

Lemma 2. Under the conditions that $\alpha>1$ and $\beta>1$, the mean deviation from the mean and the mean deviation from the median of beta-Cauchy distribution are given by

$$
E(|X-\mu|)=2 \mu G(\mu)-2 \theta G(\mu-\theta)-2 \frac{\lambda \pi^{2(\alpha+\beta-1)}(F(\mu))^{\alpha}}{\pi B(\alpha, \beta)} \sum_{i=0}^{\infty} \frac{(\beta-1)^{(i)}}{i !}(-F(\mu))^{i-1}\left[\frac{1}{\alpha+i-1}-2 \sum_{j=1}^{\infty} \frac{[F(\mu)]^{2 j} \zeta(2 j)}{(\alpha+i+2 j-1)}\right]
$$


$E(|X-m|)=\mu-2 \theta G(m-\theta)-2 \frac{\lambda \pi^{2(\alpha+\beta-1)}(F(m))^{\alpha}}{\pi B(\alpha, \beta)} \sum_{i=1}^{\infty} \frac{(\beta-1)^{(i)}}{i !}(-F(m))^{i-1}\left[\frac{1}{\alpha+i-1}-2 \sum_{j=1}^{\infty} \frac{[F(m)]^{2 j} \zeta(2 j)}{(\alpha+i+2 j-1)}\right]$,

where $\zeta$ is the Riemann zeta function defined as $\zeta(2 j)=\sum_{n=1}^{\infty} n^{-2 j}, j \geq 1$ and $(\beta-1)^{(i)}=(\beta-1)(\beta-2) \cdots(\beta-i)$ is the descending factorial.

Proof. The proof is given in the Appendix.

\subsection{Shannon's entropy}

The entropy of a random variable is a measure of variation of uncertainty. Entropy has various applications in many fields including science and engineering. For more details, see Refs. 9 and 10. Shannon's entropy is defined as $I_{S}=E(-\ln g(X))$. The following Lemma gives the Shannon's entropy for beta-Cauchy random variable.

Lemma 3. The Shannon's entropy for beta-Cauchy distribution is given by

$$
I_{S}=\ln (\lambda \pi)+\ln [B(\alpha, \beta)]-(\alpha+1)[\psi(\alpha)-\psi(\alpha+\beta)]-(\beta-1)[\psi(\beta)-\psi(\alpha+\beta)]+2 \sum_{l=1}^{\infty} \frac{(\alpha+2 l-1)^{(2 l)}}{l(\alpha+\beta+2 l-1)^{(2 l)}} \zeta(2 l),
$$

where $\psi$ is the digamma function.

Proof. By using Eq. (2), the Shannon’s entropy is given by

$$
\begin{aligned}
I_{S} & =E[-\ln g(X)] \\
& =\ln [B(\alpha, \beta)]-(\alpha-1) E[\ln F(X)]-(\beta-1) E[\ln (1-F(X))]+E\left[\ln F^{\prime}(X)\right] .
\end{aligned}
$$

By using Lemma 1 in Ref. 3, the result in Eq. (8) reduces to

$$
I_{S}=\ln [B(\alpha, \beta)]-(\alpha-1)[\psi(\alpha)-\psi(\alpha+\beta)]-(\beta-1)[\psi(\beta)-\psi(\alpha+\beta)]-E\left[\ln F^{\prime}\left(F^{-1}(Y)\right)\right],
$$

where $Y=F(x)=0.5+\pi^{-1} \arctan ((x-\theta) / \lambda)$ is the CDF of the Cauchy distribution. Now,

$$
\begin{aligned}
\ln F^{\prime}\left(F^{-1}(y)\right) & =\ln (\lambda / \pi)-\ln \left[\lambda^{2}+[\lambda \tan (\pi y-\pi / 2)]^{2}\right]=\ln (\lambda / \pi)-\ln \left[\lambda^{2} \sec ^{2}(\pi y-\pi / 2)\right] \\
& =-\ln \lambda-\ln \pi+2 \ln [\cos (\pi y-\pi / 2)]=-\ln (\lambda \pi)+2 \ln \sin (\pi y)
\end{aligned}
$$

To complete the result we need to evaluate $E(\ln \sin \pi Y)=\frac{1}{B(\alpha, \beta)} \int_{0}^{1}(\ln \sin \pi y) y^{\alpha-1}(1-y)^{\beta-1} d y$. Note that this integral is convergent for all $\alpha>0$ and $\beta>0$. By applying the Laurent series expansion of $\cot \pi z=\sum_{l=0}^{\infty} \frac{(-1)^{l} 2^{2 l} B_{2 l}(\pi z)^{2 l-1}}{(2 l) !}$, where $|z|<1$ and $B_{2 l}$ is the Bernoulli numbers ${ }^{11}$ defined as $B_{0}=1, B_{2 l}=\frac{(-1)^{l-1}(2 l) !}{2^{2 l-1} \pi^{2 l}} \zeta(2 l), l \geq 1$ and integrating the 
Laurent series of $\cot \pi z$, we have $\ln (\sin \pi z)=\ln z+\sum_{l=1}^{\infty} \frac{(-1)^{l}(2 \pi)^{2 l} B_{2 l}}{(2 l) !}\left(\frac{z^{2 l}}{2 l}\right)$. Hence,

$E(\ln \sin \pi Y)=E(\ln Y)+\sum_{l=1}^{\infty} \frac{(-1)^{l}(2 \pi)^{2 l}}{(2 l) !} \frac{B_{2 l}}{2 l} E\left(Y^{2 l}\right)$. Using the fact that $E(\ln Y)=\psi(\alpha)-\psi(\alpha+\beta),{ }^{3}$ we have

$E(\ln \sin \pi Y)=[\psi(\alpha)-\psi(\alpha+\beta)]+\sum_{l=1}^{\infty} \frac{(-1)^{l}(2 \pi)^{2 l} B_{2 l}}{(2 l) ! 2 l} \frac{B(\alpha+2 l, \beta)}{B(\alpha, \beta)}$

$$
\begin{aligned}
& =[\psi(\alpha)-\psi(\alpha+\beta)]+\sum_{l=1}^{\infty} \frac{(-1)^{l}(2 \pi)^{2 l} B_{2 l}}{(2 l) ! 2 l} \frac{\Gamma(\alpha+2 l) \Gamma(\beta)}{\Gamma(\alpha+\beta+2 l)} \frac{\Gamma(\alpha+\beta)}{\Gamma(\alpha) \Gamma(\beta)} \\
& =[\psi(\alpha)-\psi(\alpha+\beta)]+\sum_{l=1}^{\infty} \frac{(-1)^{l}(2 \pi)^{2 l} B_{2 l}}{(2 l) ! 2 l} \frac{(\alpha+2 l-1)^{(2 l)}}{(\alpha+\beta+2 l-1)^{(2 l)}},
\end{aligned}
$$

where $(\alpha)^{(k)}=\alpha(\alpha-1) \cdots(\alpha-k+1)$ is the descending factorial. Using the definition of the Bernoulli numbers, we have

$$
\begin{aligned}
E(\ln \sin \pi Y)= & {[\psi(\alpha)-\psi(\alpha+\beta)]+\sum_{l=1}^{\infty} \frac{(-1)^{l}(2 \pi)^{2 l}}{(2 l) ! 2 l} \frac{(-1)^{l-1}(2 l) !}{2^{2 l-1} \pi^{2 l}} \zeta(2 l) \frac{(\alpha+2 l-1)^{(2 l)}}{(\alpha+\beta+2 l-1)^{(2 l)}} } \\
& =[\psi(\alpha)-\psi(\alpha+\beta)]-\sum_{l=1}^{\infty} \frac{(\alpha+2 l-1)^{(2 l)}}{l(\alpha+\beta+2 l-1)^{(2 l)}} \zeta(2 l) .
\end{aligned}
$$

Hence, we obtain

$E\left[\ln F^{\prime}\left(F^{-1}(Y)\right)\right]=-\ln (\lambda \pi)+2[\psi(\alpha)-\psi(\alpha+\beta)]-2 \sum_{l=1}^{\infty} \frac{(\alpha+2 l-1)^{(2 l)}}{l(\alpha+\beta+2 l-1)^{(2 l)}} \zeta(2 l)$.

Therefore, Eq. (9) reduces to

$$
\begin{aligned}
I_{S}= & \ln [B(\alpha, \beta)]-(\alpha-1)[\psi(\alpha)-\psi(\alpha+\beta)]-(\beta-1)[\psi(\beta)-\psi(\alpha+\beta)] \\
& +\ln (\lambda \pi)-2[\psi(\alpha)-\psi(\alpha+\beta)]+2 \sum_{l=1}^{\infty} \frac{(\alpha+2 l-1)^{(2 l)}}{l(\alpha+\beta+2 l-1)^{(2 l)}} \zeta(2 l) \\
= & \ln (\lambda \pi)+\ln [B(\alpha, \beta)]-(\alpha+1)[\psi(\alpha)-\psi(\alpha+\beta)]-(\beta-1)[\psi(\beta)-\psi(\alpha+\beta)]+2 \sum_{l=1}^{\infty} \frac{(\alpha+2 l-1)^{(2 l)}}{l(\alpha+\beta+2 l-1)^{(2 l)}} \zeta(2 l) .
\end{aligned}
$$

\section{Estimation and Goodness-of-Fit Test}

Let $X_{1}, X_{2}, \ldots, X_{n}$ be a random sample from a beta-Cauchy distribution, defined in Eq. (3), with unknown parameters $\alpha, \beta, \theta$ and $\lambda$. The log-likelihood function $\ell=\ell(\alpha, \beta, \theta, \lambda)$ is given by

$$
\ell=n \ln \lambda-n \ln \pi+n(\ln \Gamma(\alpha+\beta)-\ln \Gamma(\alpha)-\ln \Gamma(\beta))+(\alpha-1) \sum_{i=1}^{n} \ln \left\{\frac{1}{2}+\frac{1}{\pi} \arctan \left(\frac{x_{i}-\theta}{\lambda}\right)\right\}
$$




$$
+(\beta-1) \sum_{i=1}^{n} \ln \left\{\frac{1}{2}-\frac{1}{\pi} \arctan \left(\frac{x_{i}-\theta}{\lambda}\right)\right\}-\sum_{i=1}^{n} \ln \left[\lambda^{2}+\left(x_{i}-\theta\right)^{2}\right]
$$

The first partial derivatives of the log-likelihood function with respect to the four parameters are

$$
\begin{aligned}
\frac{\partial \ell}{\partial \alpha}= & n(\psi(\alpha+\beta)-\psi(\alpha))+\sum_{i=1}^{n}\left\{\frac{1}{2}+\frac{1}{\pi} \arctan \left(\frac{x_{i}-\theta}{\lambda}\right)\right\}, \\
\frac{\partial \ell}{\partial \beta} & =n(\psi(\alpha+\beta)-\psi(\beta))+\sum_{i=1}^{n}\left\{\frac{1}{2}-\frac{1}{\pi} \arctan \left(\frac{x_{i}-\theta}{\lambda}\right)\right\}, \\
\frac{\partial \ell}{\partial \lambda}= & \frac{n}{\lambda}-\frac{(\alpha-1)}{\pi} \sum_{i=1}^{n}\left\{\frac{1}{2}+\frac{1}{\pi} \arctan \left(\frac{x_{i}-\theta}{\lambda}\right)\right\}^{-1}\left\{\frac{x_{i}-\theta}{\lambda^{2}+\left(x_{i}-\theta\right)^{2}}\right\} \\
& +\frac{(\beta-1)}{\pi} \sum_{i=1}^{n}\left\{\frac{1}{2}-\frac{1}{\pi} \arctan \left(\frac{x_{i}-\theta}{\lambda}\right)\right\}^{-1}\left\{\frac{x_{i}-\theta}{\lambda^{2}+\left(x_{i}-\theta\right)^{2}}\right\}-\sum_{i=1}^{n} \frac{2 \lambda}{\lambda^{2}+\left(x_{i}-\theta\right)^{2}}, \\
\frac{\partial \ell}{\partial \theta}= & \frac{(1-\alpha)}{\pi} \sum_{i=1}^{n}\left\{\frac{1}{2}+\frac{1}{\pi} \arctan \left(\frac{x_{i}-\theta}{\lambda}\right)\right\}^{-1}\left\{\frac{\lambda}{\lambda^{2}+\left(x_{i}-\theta\right)^{2}}\right\}, \\
& +\frac{(\beta-1)}{\pi} \sum_{i=1}^{n}\left\{\frac{1}{2}-\frac{1}{\pi} \arctan \left(\frac{x_{i}-\theta}{\lambda}\right)\right\}^{-1}\left\{\frac{\lambda}{\lambda^{2}+\left(x_{i}-\theta\right)^{2}}\right\}+\sum_{i=1}^{n} \frac{2\left(x_{i}-\theta\right)}{\lambda^{2}+\left(x_{i}-\theta\right)^{2}},
\end{aligned}
$$

where $\psi$ is the digamma function.

The maximum likelihood estimates (MLEs) $\hat{\alpha}, \hat{\beta}, \hat{\theta}$ and $\hat{\lambda}$ for the parameters $\alpha, \beta, \theta$, and $\lambda$, respectively, are obtained by setting the Eqs. (11) - (14) to zero and solving them simultaneously. The computations for MLEs are obtained by using the NLMIXED procedure in SAS. The standard errors for the MLEs are provided by the NLMIXED procedure. The initial estimates of $\alpha$ and $\beta$ are taken as 1 . The initial estimates for $\theta$ and $\lambda$, are taken to be the maximum likelihood estimates of $\theta$ and $\lambda$ from the Cauchy distribution by assuming the data follows the Cauchy distribution.

The likelihood ratio (LR) test can be used to test the goodness of fit of the beta-Cauchy distribution and to compare it with the Cauchy distribution. The beta-Cauchy distribution reduces to the Cauchy distribution when $\alpha=\beta=1$. Thus, to test the hypothesis

$$
H_{0}: \alpha=\beta=1 \text { against } H_{1}: H_{0} \text { is not true, }
$$

one can use the LR test by computing

$$
\delta=2[\ell(\hat{\alpha}, \hat{\beta}, \hat{\theta}, \hat{\lambda})-\ell(1,1, \tilde{\theta}, \tilde{\lambda})],
$$

where $\ell(\hat{\alpha}, \hat{\beta}, \hat{\theta}, \hat{\lambda})$ is the log-likelihood statistic for the beta-Cauchy distribution and $\ell(1,1, \tilde{\theta}, \tilde{\lambda})$ is the log-likelihood statistic for the Cauchy distribution. The statistic, $\delta$, is asymptotically distributed as chi-square with 2 degrees of freedom. 


\section{Simulation}

In this section, the relation between beta-Cauchy and beta random variables in Eq. (5) is used to simulate data from betaCauchy distribution. The maximum likelihood estimates are computed for each simulated sample. Results on the biases (estimate - actual) and the standard deviations of the estimates are used to investigate the performance of the method of maximum likelihood estimation. The simulation is carried out for various values of the parameters of the beta-Cauchy distribution and the results are similar. For brevity, we report the results for the parameter values $\alpha=0.7,1.0,4.0, \beta=$ 0.7, 1.0, 4.0, 6.0, $\lambda=2.0$ and $\theta=4.0$. Three different sample sizes of $n=250,500$, and 1000 are used in the simulation. For each sample size and each parameter combination, the maximum likelihood estimates of the parameters $\alpha, \beta, \lambda$, and $\theta$ are computed and the process is repeated 200 times. The average bias and the standard deviation of the maximum likelihood estimates are computed. The results are reported in Table 2.

Table 2. Bias (standard deviation in parentheses) of parameter estimates for $\lambda=2.0, \theta=4.0$.

\begin{tabular}{|c|c|c|c|c|c|c|}
\hline \multicolumn{3}{|c|}{ Actual values } & $\hat{\alpha}$ & $\hat{\beta}$ & $\hat{\lambda}$ & $\hat{\theta}$ \\
\hline 0.7 & 0.7 & $\begin{array}{r}250 \\
500 \\
1000\end{array}$ & $\begin{array}{l}-0.0028(0.0781) \\
-0.0090(0.0591) \\
-0.0020(0.0384)\end{array}$ & $\begin{array}{l}-0.0059(0.0822) \\
-0.0062(0.0590) \\
-0.0081(0.0425)\end{array}$ & $\begin{array}{r}0.0185(0.2859) \\
-0.0027(0.2164) \\
0.0034(0.1392)\end{array}$ & $\begin{array}{l}-0.0046(0.2557) \\
-0.0022(0.1728) \\
-0.0174(0.1253)\end{array}$ \\
\hline & 1.0 & $\begin{array}{r}250 \\
500 \\
1000\end{array}$ & $\begin{array}{l}-0.0098(0.0796) \\
-0.0050(0.0553) \\
-0.0000(0.0385)\end{array}$ & $\begin{array}{r}-0.0228(0.1199) \\
-0.0145(0.0850) \\
0.0018(0.0601)\end{array}$ & $\begin{array}{r}-0.0396(0.2874) \\
-0.0097(0.1933) \\
0.0093(0.1441)\end{array}$ & $\begin{array}{r}-0.0358(0.2717) \\
-0.0046(0.1646) \\
0.0058(0.1228)\end{array}$ \\
\hline & 4.0 & $\begin{array}{r}250 \\
500 \\
1000\end{array}$ & $\begin{array}{l}-0.0004(0.0728) \\
-0.0099(0.0512) \\
-0.0091(0.0388)\end{array}$ & $\begin{array}{l}-0.1285(0.8328) \\
-0.1564(0.6190) \\
-0.1578(0.5222)\end{array}$ & $\begin{array}{l}0.0339(0.2743) \\
0.0005(0.2004) \\
0.0080(0.1419)\end{array}$ & $\begin{array}{r}0.0174(0.3937) \\
-0.0334(0.3034) \\
-0.0389(0.2325)\end{array}$ \\
\hline & 6.0 & $\begin{array}{r}250 \\
500 \\
1000\end{array}$ & $\begin{array}{r}0.0114(0.0663) \\
-0.0047(0.0521) \\
0.0019(0.0381)\end{array}$ & $\begin{array}{r}0.1439(1.4036) \\
-0.1786(1.2266) \\
-0.0967(0.9967)\end{array}$ & $\begin{array}{r}0.0060(0.3157) \\
-0.0181(0.2481) \\
0.0157(0.1799)\end{array}$ & $\begin{array}{l}0.1538(0.4899) \\
0.0142(0.3775) \\
0.0092(0.2904)\end{array}$ \\
\hline 1.0 & 0.7 & $\begin{array}{r}250 \\
500 \\
1000\end{array}$ & $\begin{array}{l}-0.0239(0.1373) \\
-0.0164(0.0962) \\
-0.0069(0.0633)\end{array}$ & $\begin{array}{l}-0.0128(0.0785) \\
-0.0096(0.0593) \\
-0.0025(0.0391)\end{array}$ & $\begin{array}{r}0.0077(0.2811) \\
-0.0187(0.2128) \\
-0.0063(0.1369)\end{array}$ & $\begin{array}{r}0.0101(0.2631) \\
0.0196(0.1628) \\
-0.0005(0.1304)\end{array}$ \\
\hline & 1.0 & $\begin{array}{r}250 \\
500 \\
1000\end{array}$ & $\begin{array}{l}-0.0309(0.1346) \\
-0.0185(0.0898) \\
-0.0074(0.0635)\end{array}$ & $\begin{array}{l}-0.0290(0.1303) \\
-0.0147(0.0893) \\
-0.0083(0.0644)\end{array}$ & $\begin{array}{l}-0.0239(0.2978) \\
-0.0293(0.1937) \\
-0.0063(0.1401)\end{array}$ & $\begin{array}{r}-0.0052(0.2360) \\
0.0209(0.1610) \\
-0.0094(0.1183)\end{array}$ \\
\hline & 4.0 & $\begin{array}{r}250 \\
500 \\
1000\end{array}$ & $\begin{array}{l}-0.0053(0.1289) \\
-0.0109(0.0916) \\
-0.0046(0.0608)\end{array}$ & $\begin{array}{l}-0.1323(0.8156) \\
-0.1249(0.7193) \\
-0.1057(0.4978)\end{array}$ & $\begin{array}{l}0.0280(0.2906) \\
0.0116(0.1684) \\
0.0057(0.1294)\end{array}$ & $\begin{array}{l}-0.0259(0.3478) \\
-0.0102(0.3083) \\
-0.0339(0.2190)\end{array}$ \\
\hline & 6.0 & $\begin{array}{r}250 \\
500 \\
1000\end{array}$ & $\begin{array}{r}0.0123(0.1127) \\
-0.0003(0.0869) \\
-0.0009(0.0601)\end{array}$ & $\begin{array}{r}0.2001(1.3332) \\
-0.0570(1.1652) \\
-0.1149(0.8926)\end{array}$ & $\begin{array}{l}0.0130(0.2750) \\
0.0096(0.2148) \\
0.0301(0.1530)\end{array}$ & $\begin{array}{l}0.1510(0.4340) \\
0.0543(0.3270) \\
0.0030(0.2606)\end{array}$ \\
\hline 4.0 & 0.7 & $\begin{array}{r}250 \\
500 \\
1000\end{array}$ & $\begin{array}{l}-0.0271(0.8494) \\
-0.1532(0.6887) \\
-0.1563(0.5103)\end{array}$ & $\begin{array}{l}-0.0037(0.0818) \\
-0.0050(0.0552) \\
-0.0008(0.0401)\end{array}$ & $\begin{array}{r}-0.0039(0.2582) \\
0.0344(0.2101) \\
0.0448(0.1467)\end{array}$ & $\begin{array}{r}-0.0524(0.4509) \\
0.0332(0.3017) \\
0.0470(0.2202)\end{array}$ \\
\hline & 1.0 & $\begin{array}{r}250 \\
500 \\
1000\end{array}$ & $\begin{array}{l}-0.2512(0.7520) \\
-0.1220(0.6532) \\
-0.0724(0.4733)\end{array}$ & $\begin{array}{r}0.0060(0.1156) \\
-0.0249(0.0838) \\
-0.0113(0.0680)\end{array}$ & $\begin{array}{r}0.0415(0.2325) \\
-0.0033(0.1661) \\
0.0114(0.1197)\end{array}$ & $\begin{array}{r}-0.0059(0.3461) \\
0.0715(0.2642) \\
0.0393(0.2095)\end{array}$ \\
\hline
\end{tabular}


Table 2. (Continued)

\begin{tabular}{|rr|r|r|r|r|}
\hline 4.0 & 250 & $-0.0255(0.7963)$ & $-0.0712(0.7963)$ & $0.0216(0.2757)$ & $-0.0231(0.1876)$ \\
& 500 & $-0.0986(0.6874)$ & $-0.1060(0.7107)$ & $-0.0230(0.2368)$ & $0.0062(0.1502)$ \\
& 1000 & $-0.1301(0.5405)$ & $-0.1459(0.5605)$ & $-0.0402(0.1927)$ & $-0.0035(0.1004)$ \\
\hline & 250 & $0.1309(0.8121)$ & $0.2467(1.4073)$ & $0.0792(0.2861)$ & $0.0410(0.2228)$ \\
& 500 & $-0.0914(0.6806)$ & $-0.1536(1.1220)$ & $-0.0137(0.2245)$ & $-0.0063(0.1734)$ \\
& 1000 & $-0.0462(0.5747)$ & $-0.1221(1.0493)$ & $-0.0051(0.1926)$ & $-0.0109(0.1487)$ \\
\hline
\end{tabular}

The results show that the maximum likelihood estimation method performs well. In general, the estimates of the parameters and their standard deviations are reasonable. They also show that the standard deviations of the estimates decrease as the sample size increases. However, the biases, which are somewhat small, do not show a clear decreasing trend as sample size increases. For fixed $\alpha, \lambda$ and $\theta$ the bias and the standard deviation for the estimate of $\beta$ increases as $\beta$ increases. Similarly, for fixed $\beta, \lambda$ and $\theta$ the bias and the standard deviation for the estimate of $\alpha$ increases as $\alpha$ increases. The results from this study, suggest that the ML method can be used to estimate the parameters of the betaCauchy distribution.

\section{Applications of the Beta-Cauchy Distribution}

In this section, we present some applications of the beta-Cauchy distribution using three data sets to demonstrate the flexibility of the distribution to model leptokurtic, skewed (left or right) and heavy-tailed data when compared to the Cauchy and other beta family distributions.

In these applications we obtain the maximum likelihood estimates of the parameters of the fitted distributions and the values of the following statistics: AIC (Akaike Information Criterion), BIC (Bayesian Information Criterion) and AICC (Akaike Information Corrected Criterion). In addition, we compute some goodness of fit statistics in order to verify which distribution provides the best fit to the data sets. We apply Kolmogorov-Smirnov (K-S), Anderson-Darling (A-D) and Cramer-von Mises (C-M) statistics. These statistics are described in details in Refs. 12 and 13. In general, the smaller the value of these statistics, the better the fit of the data by the distribution. To conserve space, only the AIC and the K-S statistics are reported in this paper since other statistics show similar results. In addition, the empirical CDF and the CDF of any fitted model with four parameters are presented for graphical illustration of the goodness of fit.

\subsection{The Wheaton river data}

The data set on the exceedances of flood peaks $\left(\mathrm{m}^{3} / \mathrm{s}\right)$ of the Wheaton River in Yukon Territory, Canada is from Choulakian and Stephens ${ }^{14}$. They applied the generalized Pareto distribution to fit the data. Akinsete et al. ${ }^{15}$ fitted the beta-Pareto distribution to the data set.

The Cauchy and the beta-Cauchy distributions are applied to fit the data. The MLEs and the goodness of fit statistics are presented in Table 3. The results of Pareto and beta-Pareto distributions are taken from Ref. 15. Fig. 2 displays the empirical CDF and the fitted CDF's for the beta-Cauchy and beta-Pareto distributions. The goodness of fit statistics indicate that the beta-Cauchy distribution provides the best fit among these distributions since it has the smallest AIC and K-S statistics, and the largest log-likelihood value.

The beta-Cauchy distribution provides the best fit for the exceedances of flood peaks followed by the beta-Pareto distribution. The parameter estimates of $\alpha$ and $\beta$ indicate that the distribution is highly skewed to the right as $\alpha \gg \beta$. A closer look at the distribution of the data indicates the distribution is excessively skewed to the right (skewness $=1.50$ and kurtosis $=3.19$ ) with one extreme outlier. It is noted that the Cauchy distribution is not appropriate due to the skewness of the data. The Wheaton river data appears to have excessive long tail. This example suggests that the beta-Cauchy fits well a data set that is excessively right skewed with long-tailed distribution. 
Table 3. Parameter estimates (standard errors in parentheses) for the Wheaton River data

\begin{tabular}{|c|c|c|c|c|}
\hline Distribution & Pareto* & Cauchy & Beta-Pareto* & Beta-Cauchy \\
\hline $\begin{array}{l}\text { Parameter } \\
\text { Estimates }\end{array}$ & $\begin{array}{c}\hat{k}=0.2438 \\
\hat{\theta}=0.1\end{array}$ & $\begin{array}{c}\hat{\theta}=7.0718 \\
(1.4300) \\
\hat{\lambda}=6.4622 \\
(0.9825)\end{array}$ & $\begin{array}{c}\hat{\alpha}=7.6954 \\
\hat{\beta}=85.75 \\
\hat{k}=0.0208 \\
\hat{\theta}=0.1\end{array}$ & $\begin{array}{c}\hat{\alpha}=387.6492(437.7976) \\
\hat{\beta}=1.4589(0.5127) \\
\hat{\theta}=-2.0467(1.2725) \\
\hat{\lambda}=0.0787(0.0963)\end{array}$ \\
\hline K-S & 0.3185 & 0.2240 & 0.1486 & 0.1219 \\
\hline K-S $p$-value & $<0.0000$ & 0.0015 & 0.0831 & 0.2347 \\
\hline Log likelihood & -303.064 & -288.4709 & -272.1280 & -260.4802 \\
\hline AIC & 610.128 & 580.9 & 552.256 & 528.96 \\
\hline
\end{tabular}

*Parameter estimates are from Akinsete et $a l^{15}$ and no standard errors were reported.

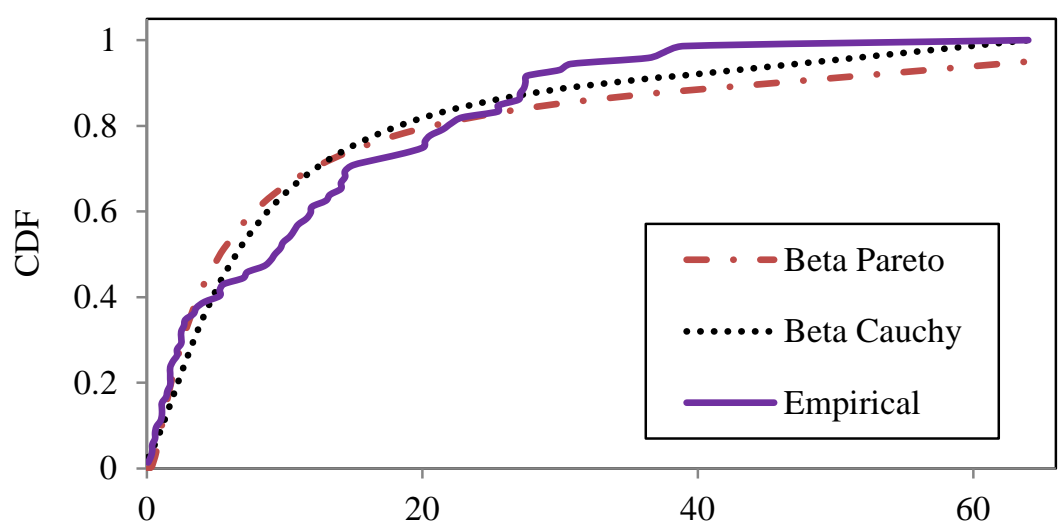

Fig. 2. CDFs for the Wheaton River data

\subsection{Strength of $1.5 \mathrm{~cm}$ glass fibres data}

In this subsection we fit the Cauchy and the beta-Cauchy distributions to the data set on the strength of the $1.5 \mathrm{~cm}$ glass fibres obtained from Ref. 16. Barreto-Souza et al. ${ }^{17}$ analyzed the data using the beta-generalized-exponential distribution. In their paper, the parameter estimates and the maximized log-likelihood values for the beta-exponential and the betageneralized exponential distributions were reported.

The beta-Cauchy distribution is applied to fit the data and compared to the beta-exponential and the beta-generalized exponential distributions. The log-likelihood, K-S, and AIC statistics are reported in Table 4.

The beta-Cauchy distribution has the largest log-likelihood value and the smallest K-S and AIC statistics. This indicates that the beta-Cauchy distribution provides a much better fit than any other distribution in Table 4. Furthermore, the adequacy of the fit is illustrated graphically in Fig. 3 by comparing the fitted CDFs with the empirical CDF. Both the beta exponential and the beta-generalized exponential distributions have lighter tails than the beta-Cauchy distribution. A closer look at the shape of the data distribution shows that the empirical data is left-skewed (skewness $=-.92$ ). This example indicates that the beta-Cauchy distribution also fits left-skewed data well, while other distributions do not. 
Table 4. Parameter estimates (standard errors in parentheses) for the glass fibres data

\begin{tabular}{|c|c|c|c|c|}
\hline Distribution & Beta-Exponential* & Cauchy & $\begin{array}{c}\text { Beta } \\
\text { Generalized Exponential* }\end{array}$ & Beta-Cauchy \\
\hline Parameter & $\hat{\alpha}=17.7786$ & $\hat{\theta}=1.5966$ & $\hat{\alpha}=0.4125$ & $\hat{\alpha}=2.4345(1.2411)$ \\
Estimates & $\hat{\beta}=22.7222$ & $\begin{array}{c}(0.0248) \\
\hat{\lambda}=0.1341 \\
(0.0235)\end{array}$ & $\hat{\beta}=93.4655$ & $\hat{\beta}=22.6124$ \\
& $\hat{\lambda}=0.3898$ & $\hat{\lambda}=0.92271$ & $\hat{\theta}=1.7053(0.0783)$ \\
& & 0.1029 & 0.1356 & $\hat{\lambda}=0.2993(0.1208)$ \\
\hline K-S & 0.1846 & 0.5167 & 0.1970 & 0.0573 \\
\hline K-S p-value & 0.0273 & -17.6365 & -15.5995 & 0.9858 \\
\hline Log likelihood & -24.1270 & 39.2730 & 39.1990 & -11.8172 \\
\hline AIC & 52.2540 & & 31.6344 \\
\hline
\end{tabular}

*Parameter estimates are from Barreto-Souza et al. ${ }^{17}$ and no standard errors were reported.

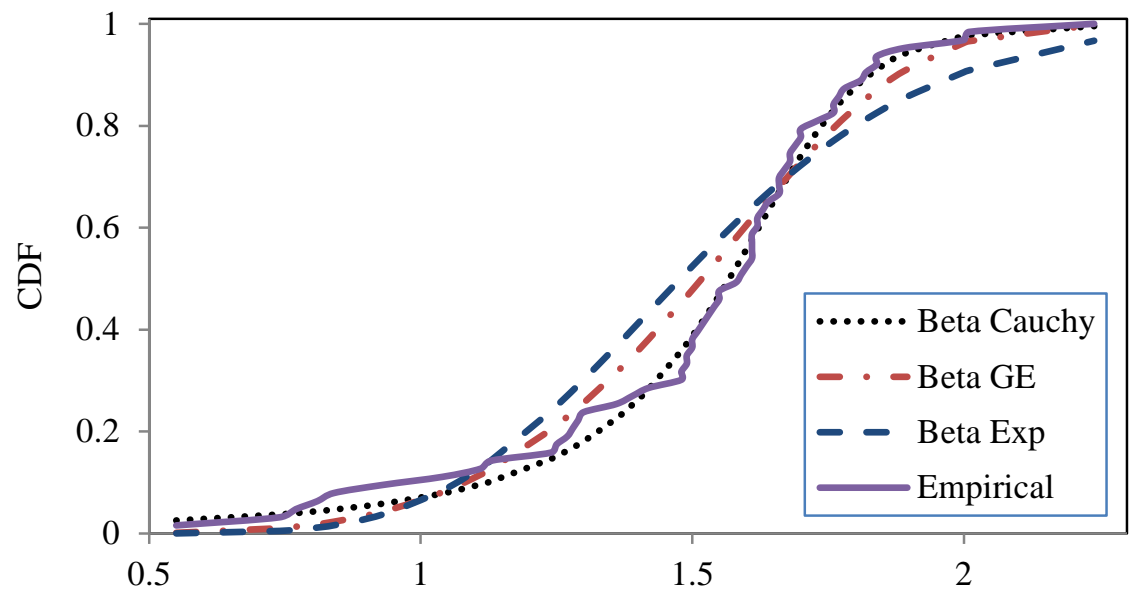

Fig. 3. CDFs for the glass fibres data

\subsection{Breaking stress of carbon fibers (GPa)}

The data set on the breaking stress of carbon fibers of $50 \mathrm{~mm}$ in length (GPa) is from Ref. 18. The Cauchy and the betaCauchy distributions are applied to fit the data. The fits are compared with Birnbaum-Saunders and beta-BirnbaumSaunders distributions studied by Cordeiro and Lemonte ${ }^{19}$. For more details about the Birnbaum-Saunders distribution and its properties, see Ref. 20.

Table 5 lists the MLEs of the parameters and the goodness of fit statistics. The MLEs of Birnbaum-Saunders and beta-Birnbaum-Saunders distributions are from Ref. 19. Fig. 4 displays the empirical CDF and the fitted CDFs for the beta-Cauchy and beta-Birnbaum-Saunders distributions.

The beta-Cauchy distribution provides a better fit to the data than the Cauchy distribution. The LR statistic for testing the null hypothesis in (15) is 17.2690 ( $p$-value 0.000178). Hence, we reject the null hypothesis in favor of the betaCauchy distribution. The beta-Cauchy distribution has the smallest K-S and AIC statistics and the largest log-likelihood value among the fitted distributions. A closer look at the distribution of the data indicates that the data set is approximately symmetric (skewness $=-.13$, and kurtosis $=.34)$ with five data values $(4.20,4.38,4.42,4.70$ and 4.90$)$ out of 66 that are relatively much larger than the next value of 3.75 . The data distribution, although approximately 
symmetric, shows an unusual shape of the distribution with some possible outliers. It appears that the beta-Cauchy distribution captures the long tail better than the beta-Birnbaum-Saunders distribution.

Table 5. Parameter estimates (standard errors in parentheses) for the carbon fibers data

\begin{tabular}{|c|c|c|c|c|}
\hline Distribution & Birnbaum-Saunders & Cauchy & $\begin{array}{c}\text { Beta } \\
\text { Birnbaum-Saunders }\end{array}$ & Beta-Cauchy \\
\hline $\begin{array}{l}\text { Parameter } \\
\text { Estimates }\end{array}$ & $\begin{array}{c}\hat{a}=0.4371 \\
(0.0381) \\
\hat{b}=2.5154 \\
(0.1321)\end{array}$ & $\begin{array}{c}\hat{\theta}=2.8604 \\
(0.0918) \\
\hat{\lambda}=0.4788 \\
(0.0776)\end{array}$ & $\begin{array}{l}\hat{\alpha}=0.1930(0.0259) \\
\hat{\beta}=1876.73(605.1) \\
\hat{a}=1.0445(0.0036) \\
\hat{b}=57.600(0.3313)\end{array}$ & $\begin{array}{c}\hat{\alpha}=13.204(25.906) \\
\hat{\beta}=15.340(31.031) \\
\hat{\theta}=3.117(0.885) \\
\hat{\lambda}=2.767(3.098)\end{array}$ \\
\hline K-S & 0.1731 & 0.0814 & 0.1271 & 0.0637 \\
\hline K-S p-value & 0.0383 & 0.7742 & 0.2369 & 0.9515 \\
\hline Log likelihood & -100.19 & -93.9693 & -91.355 & -85.3347 \\
\hline AIC & 204.38 & 191.9 & 190.71 & 178.7 \\
\hline
\end{tabular}

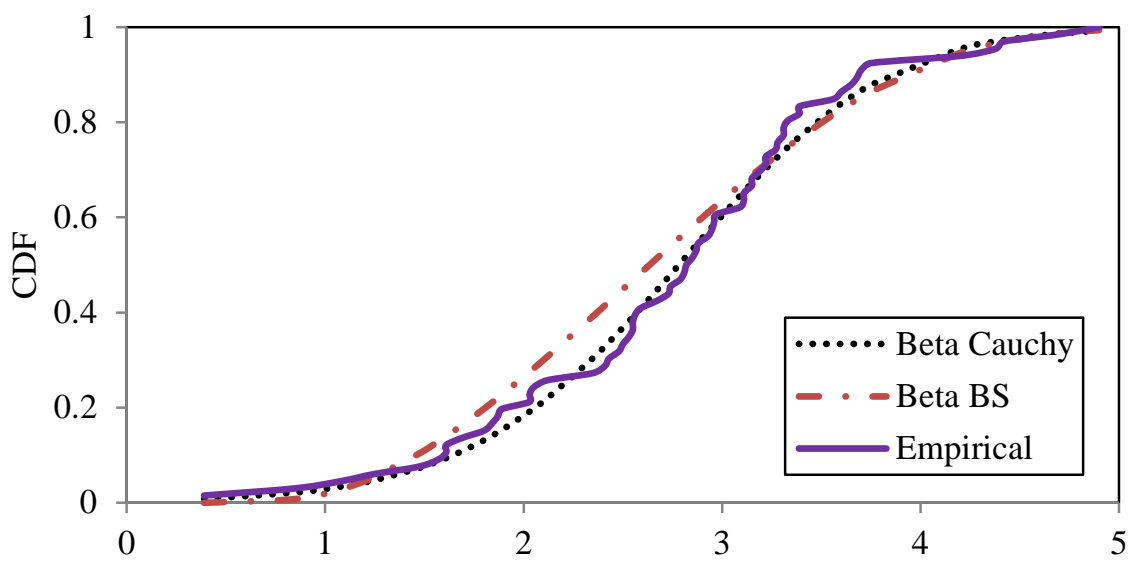

Fig. 4. CDFs for the carbon fibers data

\section{Conclusions}

In this paper, we study some properties of the beta-Cauchy distribution, including the quantile function, the mean deviations from the mean and from the median, and the Shannon's entropy. The maximum likelihood estimation method is applied to estimate the parameters of the beta-Cauchy distribution. A simulation study is conducted to investigate the performance of the MLE for different parameter values and different sample sizes. The simulation showed that the MLE performed well for finite samples. Finally, applications to real data sets are given to illustrate the flexibility of the betaCauchy distribution for fitting a variety of skewed (left or right), and long-tailed as well as high peak (leptokurtic) data sets. These applications indicate that the beta-Cauchy distribution provides more adequate fit to these data sets than other four parameter beta family members such as the beta-Birnbaum-Saunders and the beta-Pareto distributions. These applications suggest that the beta-Cauchy distribution can be a good model to fit skewed data with heavy tails. We do not have an example of platykurtic distribution. However, we anticipate that the beta-Cauchy distribution will also fit well for such type of distributions. 


\section{Appendix}

\section{Proof of Lemma 2.}

The condition $\alpha>1$ and $\beta>1$ is needed for the existence of the mean of beta-Cauchy distribution [see Theorem 3 in Ref. 5]. Now, in order to evaluate the mean deviations we need to evaluate the following integral

$$
I(a)=\int_{-\infty}^{a} x g(x) d x=\frac{1}{B(\alpha, \beta)} \int_{-\infty}^{a} x\left(\frac{1}{2}+\frac{1}{\pi} \arctan \left(\frac{x-\theta}{\lambda}\right)\right)^{\alpha-1}\left(\frac{1}{2}-\frac{1}{\pi} \arctan \left(\frac{x-\theta}{\lambda}\right)\right)^{\beta-1} \frac{\lambda}{\pi} \frac{d x}{(x-\theta)^{2}+\lambda^{2}}
$$

Let $t=x-\theta$. Then,

$$
\begin{gathered}
I(a)=\frac{\lambda}{\pi B(\alpha, \beta)} \int_{-\infty}^{a-\theta}(\theta+t)\left(\frac{1}{2}+\frac{1}{\pi} \arctan \left(\frac{t}{\lambda}\right)\right)^{\alpha-1}\left(\frac{1}{2}-\frac{1}{\pi} \arctan \left(\frac{t}{\lambda}\right)\right)^{\beta-1} \frac{d t}{t^{2}+\lambda^{2}} \\
=\frac{\lambda \theta}{\pi B(\alpha, \beta)} \int_{-\infty}^{a-\theta}\left(\frac{1}{2}+\frac{1}{\pi} \arctan \left(\frac{t}{\lambda}\right)\right)^{\alpha-1}\left(\frac{1}{2}-\frac{1}{\pi} \arctan \left(\frac{t}{\lambda}\right)\right)^{\beta-1} \frac{d t}{t^{2}+\lambda^{2}} \\
\quad+\frac{\lambda}{\pi B(\alpha, \beta)} \int_{-\infty}^{a-\theta} t\left(\frac{1}{2}+\frac{1}{\pi} \arctan \left(\frac{t}{\lambda}\right)\right)^{\alpha-1}\left(\frac{1}{2}-\frac{1}{\pi} \arctan \left(\frac{t}{\lambda}\right)\right)^{\beta-1} \frac{d t}{t^{2}+\lambda^{2}} \\
=\theta G(a-\theta)+\frac{\lambda \pi^{\alpha+\beta-1}}{B(\alpha, \beta)} \int_{-\infty}^{a-\theta} t\left(\frac{\pi}{2}+\arctan \left(\frac{t}{\lambda}\right)\right)^{\alpha-1}\left(\frac{\pi}{2}-\arctan \left(\frac{t}{\lambda}\right)\right)^{\beta-1} \frac{d t}{t^{2}+\lambda^{2}} .
\end{gathered}
$$

Let $u=(\pi / 2)+\arctan (t / \lambda)$. Then, $t=\lambda \tan (u-(\pi / 2))=-\lambda \cot u$ and $d t=\lambda \csc ^{2} u d u$, the integral

$$
\int_{-\infty}^{a-\theta} t\left(\frac{\pi}{2}+\arctan \left(\frac{t}{\lambda}\right)\right)^{\alpha-1}\left(\frac{\pi}{2}-\arctan \left(\frac{t}{\lambda}\right)\right)^{\beta-1} \frac{d t}{t^{2}+\lambda^{2}}=-\int_{0}^{\pi F(a)} \cot u u^{\alpha-1}(\pi-u)^{\beta-1} d u
$$

where $F(a)$ is the Cauchy CDF evaluated at $a$.

By using the generalized binomial expansion

$$
(\pi-u)^{\beta-1}=\pi^{\beta-1} \sum_{i=0}^{\infty} \frac{(\beta-1)^{(i)}}{i !}(-1)^{i}\left(\frac{u}{\pi}\right)^{i}=\sum_{i=0}^{\infty} \frac{(\beta-1)^{(i)}}{i !}(-1)^{i} u^{i} \pi^{\beta-i-1},
$$

where $(\beta-1)^{(i)}=(\beta-1)(\beta-2) \cdots(\beta-i)$ is the descending factorial, we evaluate the integral in Eq. (A.1) to obtain

$$
-\int_{0}^{\pi F(a)} \cot u u^{\alpha-1}(\pi-u)^{\beta-1} d u=\sum_{i=0}^{\infty} \frac{(\beta-1)^{(i)}}{i !}(-1)^{i+1} \pi^{\beta-i-1} \int_{0}^{\pi F(a)} u^{\alpha+i-1} \cot u d u .
$$

Using the Laurent series expansion of $\cot u$ (Ref. 11), Eq. (A.1) reduces to

$$
\begin{aligned}
\sum_{i=0}^{\infty} \frac{(\beta-1)^{(i)}}{i !}(-1)^{i+1} \pi^{\beta-i-1}\left\{\sum_{j=0}^{\infty} \frac{(-1)^{j} 2^{2 j} B_{2 j}[\pi F(a)]^{\alpha+i-1+2 j}}{(\alpha+i-1+2 j)(2 j) !}\right\} \\
=\sum_{i=0}^{\infty} \frac{(\beta-1)^{(i)}}{i !}(-1)^{i+1} \pi^{\beta-i-1}\left\{\frac{(\pi F(a))^{\alpha+i-1}}{\alpha+i-1}+\sum_{j=1}^{\infty} \frac{(-1)^{j} 2^{2 j} B_{2 j}[\pi F(a)]^{\alpha+i+2 j-1}}{(\alpha+i+2 j-1)(2 j) !}\right\} .
\end{aligned}
$$

Using the definition of Bernoulli numbers, we have 
$\sum_{j=1}^{\infty} \frac{(-1)^{j} 2^{2 j} B_{2 j}[\pi F(a)]^{\alpha+i+2 j-1}}{(\alpha+i+2 j-1)(2 j) !}=\sum_{j=1}^{\infty} \frac{(-1)^{j} 2^{2 j}[\pi F(a)]^{\alpha+i+2 j-1}}{(\alpha+i+2 j-1)(2 j) !} \frac{(-1)^{j-1}(2 j) !}{2^{2 j-1} \pi^{2 j}} \zeta(2 j)=-2 \sum_{j=1}^{\infty} \frac{[\pi F(a)]^{\alpha+i+2 j-1} \zeta(2 j)}{(\alpha+i+2 j-1) \pi^{2 j}}$.

Thus, we have

$$
\begin{aligned}
& \int_{-\infty}^{a-\theta} t\left(\frac{\pi}{2}+\arctan \left(\frac{t}{\lambda}\right)\right)^{\alpha-1}\left(\frac{\pi}{2}-\arctan \left(\frac{t}{\lambda}\right)\right)^{\beta-1} \frac{d t}{t^{2}+\lambda^{2}} \\
& =\sum_{i=0}^{\infty} \frac{(\beta-1)^{(i)}}{i !}(-1)^{i+1} \pi^{\beta-i+1}\left\{\frac{(\pi F(a))^{\alpha+i-1}}{\alpha+i-1}-2 \sum_{j=1}^{\infty} \frac{[\pi F(a)]^{\alpha+i+2 j-1} \zeta(2 j)}{(\alpha+i+2 j-1) \pi^{2 j}}\right\} .
\end{aligned}
$$

Therefore,

$$
I(a)=\theta G(a-\theta)+\frac{\lambda \pi^{2(\alpha+\beta-1)}(F(a))^{\alpha}}{\pi B(\alpha, \beta)} \sum_{i=0}^{\infty} \frac{(\beta-1)^{(i)}}{i !}(-F(a))^{i-1}\left[\frac{1}{\alpha+i-1}-2 \sum_{j=1}^{\infty} \frac{[F(a)]^{2 j} \zeta(2 j)}{(\alpha+i+2 j-1)}\right] .
$$

The proof is complete by applying $I(\mu)$ and $I(m)$ to Eqs. (6) and (7), respectively.

\section{References:}

1. N. Eugene, C. Lee and F. Famoye, Beta-normal distribution and its applications, Communications in Statistics-Theory and Methods, 31, (2002) 497-512.

2. M.C. Jones, Families of distributions arising from distributions of order statistics, Test, 13, (2004) 1-43.

3. K. Zografos and N. Balakrishnan, On families of beta- and generalized gamma-generated distributions and associated inference, Statistical Methodology, 6, (2009) 344-362.

4. C. Lee, F. Famoye and A. Alzaatreh, Methods for generating families of univariate continuous distributions in the recent decades, WIREs Computational Statistics, 5, (2013) 219-238.

5. E. Alshawarbeh, C. Lee and F. Famoye, The beta-Cauchy distribution, Journal of Probability and Statistical Science, 10(1), (2012) 41-57.

6. G. Steinbrecher and W.T. Shaw, Quantile mechanics, European Journal of Applied Mathematics, 19, (2008) 87-112.

7. F. Galton, Enquiries into Human Faculty and its Development (Macmillan \& Company, London, 1883).

8. J.J. Moors, A quantile alternative for kurtosis, The Statistician, 37, (1988) 25-32.

9. J.N. Kapur, Measures of Information and Their Applications (John Wiley \& Sons, New York, 1994).

10. C. Arndt, Information Measures: Information and its Description in Science and Engineering (Springer, Berlin, 2001).

11. I.S., Gradshteyn and I.M. Ryzhik, Table of Integrals, Series, and Products, (Edited by Jeffrey, A. and Zwillinger, D.) $7^{\text {th }}$ Edition (Academic Press, San Diego, 2007).

12. G. Chen and N. Balakrishnan, A general purpose approximate goodness of fit test, Journal of Quality Technology, 27, (1995) 154-161.

13. R.B. D’Agostino and M.A. Stephens, Goodness of Fit Techniques (Marcel Dekker, New York, 1986).

14. V. Choulakian and M.A. Stephens, Goodness-of-fit for the generalized Pareto distribution, Technometrics, 43(4), (2001) 478484.

15. A. Akinsete, F. Famoye and C. Lee, The beta-Pareto distribution, Statistics, 42(6), (2008) 547-563.

16. R.L. Smith and J.C. Naylor, A comparison of maximum likelihood and Bayesian estimators for the three-parameter Weibull distribution, Applied Statistics, 36, (1987) 358-369.

17. W. Barreto-Souza, A.H.S. Santos and G.M. Cordeiro, The beta generalized exponential distribution, Journal of Statistical Computation and Simulation, 80(2), (2010) 159-172.

18. M.D. Nichols and W.J. Padgett, A bootstrap control chart for Weibull percentiles, Quality and Reliability Engineering International, 22, (2006) 141-151.

19. G.M. Cordeiro and A.J. Lemonte, The $\beta$-Birnbaum-Saunders distribution: An improved distribution for fatigue life modeling, Computational Statistics and Data Analysis, 55(3), (2011) 1445-1461.

20. Z.W. Birnbaum, and S.C. Saunders, A new family of life distributions, Journal of Applied Probability, 6, (1969) 319-327. 